\title{
Dimensões ambientais e produtos e tecnologias influentes na participação e inclusão de alunos com incapacidade
}

\section{Environmental Dimensions and Products and Technology that Influence Participation and Inclusion of Students with Disabilities}

\author{
Mónica Silveira-Maia*1 \\ msmaia@ese.ipp.pt \\ Pedro Lopes-Dos-Santos** \\ plsantos.fpce@gmail.com \\ Manuela Sanches-Ferreira* \\ manuelaferreira@ese.ipp.pt \\ Silvia Alves* \\ alvessilvia33@gmail.com \\ AnA PARADA*** \\ ana.parada@usc.es \\ *Politécnico do Porto, Portugal \\ **Universidade do Porto, Portugal \\ ***Universidade de Santiago de Compostela, España
}

\section{Resumo:}

Este artigo teve como objetivo identificar aspetos ambientais influentes no processo de inclusão de alunos com incapacidade. Foram analisados Programas Educativos Individuais (PEI) de 170 alunos com necessidades adicionais de suporte, num processo de recolha que cobriu diferentes regiões de Portugal Continental. Os conteúdos dos PEI foram objeto de análise de conteúdo, procedendo-se à identificação de unidades de significado e sua categorização dedutiva nas taxonomias de: Whiteneck et al. (1997)

\begin{abstract}
:
The aim of this study was to identify environmental conditions that influence the inclusion process of students with disabilities. To this end, the Individualized Education Programs (IEP) for 170 students with additional support needs were analyzed. The sample represented all regions of continental Portugal. The IEP were subject to content analysis with the identification of meaning units and its deductive categorization within the taxonomies proposed by Whiteneck et al. (1997) - accessibility,
\end{abstract}

1 Dirección para correspondencia (correspondence address):

Mónica Silveira-Maia. Rua Dr. Roberto Frias, 602. 4200-465 Porto (Portugal). 
Dimensões ambientais e produtos e tecnologias influentes na participação e inclusão de alunos com incapacidade

Mónica Silveira-Maia, Pedro lopes-dos-Santos, Manuela Sanches-Ferreira, Silvia Alves y

ANA PARADA

- acessibilidade, disponibilidade, adaptabilidade, suporte social e equidade; e da CIFCJ (OMS, 2007) - produtos e tecnologias, ambiente natural, apoio e relacionamentos, atitudes e serviços, sistemas e politicas. Os resultados sugerem que o suporte social (e.g., atitudes, comportamentos de apoio), a disponibilidade (e.g., serviços, recursos, materiais) e a adaptabilidade (e.g., ajustamento de métodos/ tarefas/ materiais) são, na globalidade dos casos, as dimensões mais consideradas no apoio à participação dos alunos com incapacidade. De modo correspondente, os domínios da CIF-CJ mais abordados foram o apoio e relacionamentos (i.e., apoio prático físico ou emocional) e os produtos e tecnologias, especificamente métodos/ materiais educativos especialmente concebidos ou adaptados. A partir destes resultados são discutidas implicações práticas em diferentes níveis do sistema educativo.

\section{Palavras chave:}

Participação educacional; inclusão; necessidades adicionais de suporte; fatores ambientais; produtos e tecnologias; CIFCJ; PEI.

availability, adaptability, social support and equity - and the ICF-CY (WHO, 2007) - products and technology, natural environment, support and relationships, attitudes, and services, systems and policies. The results suggested that social support (e.g., attitudes, supportive behaviors) and availability (e.g., services, resources, materials) and adaptability (e.g., adjustment of methods/tasks/materials) were the most referenced dimensions in the support provided to enhance students' participation. In a congruent way, the most mentioned domains of the ICF-CY were support and relationships (i.e., physical and emotional practical support) and products and technology, specifically educational methods/ materials adapted or especially designed. Based on these results practical implications on different educational system levels were outlined.

\section{Key-words:}

Educational participation; inclusion; additional support needs; environmental factors; products and technology; ICF-CY; IEP.

\section{Resumé:}

Cet article vise l'identification de plusieurs variables de nature environnementale qui influencent le processus d'inclusion des étudiants handicapés. On a analysé les programmes éducatifs individuels (PEI) de 170 élèves ayant des besoins d'assistance supplémentaires, en suivant une procédure d'échantillonnage qui couvrait différentes régions du Portugal continental. Le contenu de chaque PEI a été l'objet d'une analyse de contenu où étaient identifiées des unités de signification qui étaient après catégorisées déductivement en utilisant des catégories incluses dans les taxonomies de Whiteneck et al. (1997) - accessibilité, disponibilité, adaptabilité, soutien social et équité - et de la CIF-EA (OMS, 2007) - produits et systèmes techniques, environnement naturel, soutiens et relations, les attitudes et services, systèmes et politiques. Les résultats suggèrent que, dans I'ensemble des cas, le soutien social, la disponibilité et l'adaptabilité sont les dimensions plus considérées dans le soutien à la participation des étudiants handicapés. Corrélativement, les domaines plus considérés de la CIF-EA ont été les soutiens et relations et les produits et systèmes techniques, en particulier les méthodes / matériels pédagogiques spécialement conçus ou adaptés. Ces résultats sont discutés en termes de ses implications pratiques aux différents niveaux d l'enseignement.

\section{Mots clés:}

Participation dans l'éducation ; inclusion; besoins de assistance supplémentaires; facteurs environnementaux; produits et systèmes techniques; CIF-EA; PEI.

Fecha de recepción: 16-5-2017

Fecha de aceptación: 14-9-2017 
Mónica Silveira-Maia, Pedro Lopes-dos-Santos, Manuela Sanches-Ferreira, Silvia Alves y

\section{Introdução}

Inscrita nos valores de uma sociedade democrática, a inclusão integra na sua definição uma educação de qualidade para todos os alunos - incluindo aqueles que experienciam algum tipo de incapacidade -, visando a sua autonomia e acesso pleno à cidadania (e.g., Norwich, 2014; Terzi, 2014). Sublinhando o respeito e celebração da diversidade humana, a educação inclusiva é hoje compreendida como um processo que visa uma igualdade que não se cinge ao acesso e partilha de espaços comuns de aprendizagem, mas compreende o desenvolvimento e apropriação de oportunidades para uma participação e envolvimento bem-sucedido por parte de todos (e.g., Florian, 2014; EADSNE, 2011; Sanches-Ferreira et al., 2013). Tal desígnio implica concretizações várias que devem refletir, conforme enunciado pela Agência Europeia EADSNE, em Development of a set of indicators - for inclusive education in Europe (Kyriazopoulou \& Weber, 2009), a mobilização de diferentes níveis do sistema educativo, desde aspetos macro como são quadros legais, políticos e administrativos, até os níveis meso e micro, que incluem, respetivamente, os serviços da escola e comunidade, e o contexto de sala de aula.

A nortear a atuação destes três níveis do sistema, tem estado um racional de inclusão onde, segundo Reindal (2010), se pode distinguir uma vertente ética e sociopolítica e outra de cariz ontológica e epistemológica. Os argumentos construídos no plano ético e sociopolítico enfatizam os conceitos de democracia, equidade e direitos, global e consensualmente plasmados nos articulados legais definidores do macro sistema em educação. Já a transladação desses conceitos para o domínio das práticas - sobretudo, a nível meso e micro sistémico - tem sido alvo de maior discussão, refletindo inquietações nos planos ontológico e epistemológico sobre questões como o entendimento de deficiência e incapacidade e sobre a existência (ou não) de reais diferenças entre alunos com e sem incapacidade. De facto, ao prevalecer um entendimento da diferença e da incapacidade como constructos sociais, realçando a inexistência - ontologicamente real - de uma diferenciação clara entre aqueles com e sem incapacidade, o desenvolvimento de respostas diferentes para esses dois, supostamente distintos, grupos é questionada e alvo de atual desconstrução (Reindal, 2010).

Conforme é defendido na abordagem de capacitação, desenvolvida por Amartya Sen (1992), a diferença constitui uma "variável especifica 
Dimensões ambientais e produtos e tecnologias influentes na participação e inclusão de alunos com incapacidade

Mónica Silveira-Maia, Pedro lopes-dos-Santos, Manuela Sanches-Ferreira, Silvia Alves y ANA PARADA

numa realidade objetiva que deve ser avaliada tendo por referência as funcionalidades e capacidades do individuo" (Terzi 2004, p. 155). Esta abordagem transparece uma perspetiva biopsicossocial onde a incapacidade é entendida como resultado de uma interação negativa "entre um indivíduo e seus fatores contextuais" (OMS, 2001, p. 186). Ideia também vertida pela perspetiva de ajustamento individuo-meio proposta por Shalock et al. (2010), que define a incapacidade como um desalinhamento entre as necessidades, capacidades e aspirações do indivíduo e os recursos, exigências e oportunidades promovidos pelo meio (Shogren et al., 2016). Subjacente a este entendimento está a premissa de que se o ambiente for desenhado para acolher um largo espectro de diversidade funcional, encontrando-se munido de suportes adequados, os indivíduos poderão participar ativamente na sociedade. À incapacidade e funcionalidade foi, deste modo, atribuído um caracter situacional determinado por variáveis ambientais.

O significado operacional de inclusão tem sido, por isso, inscrito no potencial do meio para responder à diversidade de necessidades dos alunos, identificando e removendo barreiras à sua efetiva e continua participação (e.g., Maxwell \& Granlund, 2011).

Não obstante a variedade de estudos que mostram a influencia determinante do meio sobre a funcionalidade, a participação social e a qualidade de vida dos indivíduos com incapacidade (e.g., Rosenberg, Ratzon, Jarus \& Bart, 2011), o ambiente representa um conceito ainda difícil de definir e avaliar. De facto, conforme argumenta Noreau e Boschen (2010), o modo tão complexo e individualizado como o ambiente intersecta a participação de cada sujeito tem inviabilizado a sua descrição clara e objetiva, repercutindo-se naturalmente na identificação e sistematização de variáveis influentes no processo de inclusão. Esta aparente indissociabilidade entre os conceitos ambiente e a participação, era já sugerida na década de 40 do século XX por Lewin, quando propôs a equação $B=f(P, E)$, para explicar que o comportamento humano (B) é função da pessoa $(P)$ e do meio (E). Na sua teoria de campo, Lewin (1935) lança três premissas que colocam em evidência a complexidade inerente à análise da influencia ambiental sobre o comportamento humano: (i) as situações devem ser apreendidas de modo holístico e não dissecadas em partes; (ii) o mesmo ambiente pode desencadear comportamentos diferentes em duas pessoas; e (iii) ambientes diferentes podem obter respostas similares de outras duas. A par do modelo ecológico do 
Mónica Silveira-Maia, Pedro lopes-dos-Santos, Manuela Sanches-Ferreira, Silvia Alves y

desenvolvimento (Process-Person-Context-Time Model) proposto, posteriormente, por Bronfenbrenner (1979), Lewin (1935) foi um percursor chave de subsequentes conceptualizações e taxonomias sobre o meio, deixando claro que o ambiente é bem mais do que os seus aspetos físicos. Assim, objeto de vários exercícios de categorização, é hoje global e grosseiramente consensual que o ambiente refere-se a fatores extrínsecos capazes de influenciar o comportamento e que engloba os contextos: físico, social/atitudinal, politico e natural (e.g., OMS, 2001; Fougeyrollas \& Beauregard, 2001).

Um dos mais citados ensaios de classificação da influencia do meio sobre o comportamento humano, é a proposta por Whiteneck, Fougeyrolles e Gerhart (1997), onde as características ambientais surgem agrupadas em cinco dimensões: acessibilidade, adaptabilidade, disponibilidade, suporte social e equidade (tabela $\mathbf{1}$ ).

\section{Tabela 1. Dimensões de impacte ambiental propostas por Whiteneck et al.} (1997).

\begin{tabular}{|c|c|c|}
\hline Dimensão & Questão chave & Explicação \\
\hline & $\begin{array}{l}\text { É tratado igualmente } \\
\text { com os outros? } \\
\text { /are you treated } \\
\text { equally with others?/ }\end{array}$ & $\begin{array}{l}\text { Aspetos políticos/ } \\
\text { institucionais que ga- } \\
\text { rantem a igualdade } \\
\text { de oportunidades. } \\
\end{array}$ \\
\hline & $\begin{array}{l}\text { É aceite e apoiado } \\
\text { pelos outros? } \\
\text { /are you accepted by } \\
\text { those around you? I }\end{array}$ & $\begin{array}{l}\text { Aspetos atitudinais } \\
\text { que desencorajam ou } \\
\text { facilitam a integração } \\
\text { na comunidade. }\end{array}$ \\
\hline & $\begin{array}{l}\text { As suas necessidades } \\
\text { são atendidas/corres- } \\
\text { pondidas? } \\
\text { /are your special ne- } \\
\text { eds met?/ }\end{array}$ & $\begin{array}{l}\text { Aspetos relativos à } \\
\text { existência de ser- } \\
\text { viços e recursos ne- } \\
\text { cessários ao indivi- } \\
\text { duo. }\end{array}$ \\
\hline & $\begin{array}{l}\text { Consegue fazer aqui- } \\
\text { lo que quer? } \\
\text { /can you do what } \\
\text { you want to do?/ }\end{array}$ & $\begin{array}{l}\text { Aspetos à adequação } \\
\text { dos equipamentos/ } \\
\text { tarefas às necessida- } \\
\text { des do individuo }\end{array}$ \\
\hline & $\begin{array}{l}\text { Consegue ir onde } \\
\text { quer? } \\
\text { /can you get to whe- } \\
\text { re you want to go?/ }\end{array}$ & $\begin{array}{l}\text { Aspetos físicos que } \\
\text { restringem ou facili- } \\
\text { tam a possibilidade } \\
\text { do individuo mover- } \\
\text { se livremente na co- } \\
\text { munidade }\end{array}$ \\
\hline
\end{tabular}


Dimensões ambientais e produtos e tecnologias influentes na participação e inclusão de alunos com incapacidade

Mónica Silveira-Maia, Pedro lopes-dos-Santos, Manuela Sanches-Ferreira, Silvia Alves y ANA PARADA

Esta classificação toma como unidade de análise o meio e o tipo de interação que estabelece com o individuo, referindo-se à liberdade para entrar, aproximar-se, comunicar e fazer parte de uma situação, isto é, a liberdade para participar.

Mais recentemente, o crescente interesse no escrutínio de aspetos ambientais influentes na funcionalidade e inclusão vê-se refletido na Classificação Internacional de Funcionalidade, Incapacidade e Saúde (CIF) proposta pela Organização Mundial de Saúde (OMS, 2001). Representando um referencial teórico que permite pensar a incapacidade e funcionalidade sob uma perspetiva biopsicossocial, a CIF oferece uma taxonomia organizadora das variáveis influentes na funcionalidade humana que são globalmente agrupadas em três componentes: Funções e Estruturas do Corpo, Atividades e Participação e Fatores Ambientais. Na sistematização dos fatores ambientais podem-se distinguir nesta classificação dois níveis conceptuais: um individual referindo-se a aspetos físicos/ materiais e relacionais do ambiente próximo; e outro social que inclui serviços e sistemas formais e informais na comunidade. Em termos estruturais, os fatores ambientais são listados em torno de cinco domínios (tabela 2), compostos por um conjunto mais detalhado de categorias ambientais que, em interação com as características especificas de cada sujeito (i.e., Funções e Estruturas do Corpo), se podem revelar facilitadores ou barreiras ao seu envolvimento nas Atividades e Participação.

Tabela 2. Fatores Ambientais representados na CIF (OMS, 2001, 154-170).

\begin{tabular}{|c|c|}
\hline Domínios & Descrição \\
\hline $\begin{array}{l}\text { Produtos e } \\
\text { Tecnologias }\end{array}$ & $\begin{array}{l}\text { "Produtos naturais ou fabricados pelo homem ou sistemas de } \\
\text { produtos, equipamentos e tecnologias existentes no ambiente } \\
\text { imediato do indivíduo" (e.g., alimentos, medicamentos, aspetos } \\
\text { arquitetónicos, brinquedos educativos, softwares adaptados, } \\
\text { sistemas aumentativos e alternativos de comunicação, domótica, } \\
\text { dispositivos para a mobilidade) }\end{array}$ \\
\hline $\begin{array}{l}\text { Ambiente } \\
\text { Natural }\end{array}$ & $\begin{array}{l}\text { "Elementos animados e inanimados do ambiente natural ou físi- } \\
\text { co, e dos componentes deste ambiente que foram modificados } \\
\text { pelas pessoas" (e.g., temperatura, luz, som, vibração) }\end{array}$ \\
\hline $\begin{array}{l}\text { Apoio e } \\
\text { Relaciona- } \\
\text { mentos }\end{array}$ & $\begin{array}{l}\text { "Pessoas ou animais que dão apoio prático físico ou emocional, } \\
\text { assim como na educação, proteção e assistência, e nos relacio- } \\
\text { namentos com outras pessoas" (e.g., apoio da família próxima e } \\
\text { alargada, dos pares, amigos, profissionais) }\end{array}$ \\
\hline
\end{tabular}


Mónica Silveira-Maia, Pedro Lopes-dos-Santos, Manuela Sanches-Ferreira, Silvia Alves y

\begin{tabular}{ll}
\hline Atitudes & "Atitudes individuais ou da sociedade sobre a confiança, mere- \\
& $\begin{array}{l}\text { cimento e valor de um ser humano que podem motivar práti- } \\
\text { cas positivas e honrosas ou negativas e discriminatórias" (e.g., } \\
\text { atitudes individuais da família próxima, da família alargada, de } \\
\text { amigos, de pares, de conhecidos) }\end{array}$ \\
\hline Serviços, & "Programas estruturados e operações (...) mecanismos de contro- \\
Sistemas e & lo administrativo e de supervisão organizativa (...) regras, regula- \\
Politicas & $\begin{array}{l}\text { mentos, convenções e normas (...) organizados para satisfazer as } \\
\text { necessidades dos indivíduos" (e.g., serviços, sistemas e politicas } \\
\text { de utilidade publica, de transportes, de proteção civil, de segu- } \\
\text { rança social, de educação) }\end{array}$ \\
\hline
\end{tabular}

A CIF, especificamente a sua versão adaptada a crianças e jovens (CIFCJ -OMS, 2007), tem sido apontada como um modelo útil ao desenvolvimento de respostas inclusivas em contexto educativo, por se reconhecer na sua utilização a possibilidade de (i) a nível individual, promover uma caracterização holística e dinâmica do funcionamento do aluno informando; (ii) a nível microssistémico, o delineamento de suportes a implementar na sala de aula (Simeonsson, Simeonsson \& Hollenweger, 2008); e por, (iii) a nível global, poder-se antever a possibilidade de sistematizar / inventariar variáveis influentes no processo de inclusão que mereçam ser consideradas a nível meso e macrossistémico na organização das respostas educativas - desde o desenho dos projetos educativos (ao nível da escola), até a aspetos políticos e administrativos que visem a mobilização de sistemas de suporte (e.g., Hollenweger, 2011).

Estas expectativas geradas em torno da utilização da CIF-CJ - usada de forma pioneira em alguns Países como é o caso de Portugal (DecretoLei n. ${ }^{0}$ 3/2008) -, não encontram, por ora, correspondência no plano instrumental e prático, onde escasseiam ferramentas de avaliação focadas na mensuração / registo de fatores do ambiente. Essa é uma das razões apontadas na literatura para praticas educacionais ainda pouco focadas na habilitação ambiental (e.g., Silveira-Maia, Lopes-dos-Santos \& Sanches-Ferreira, 2016; Sanches-Ferreira et al., 2015), onde a manipulação de variáveis ambientais (e.g., estratégias, materiais) é ainda feita de modo intuitivo, pouco sistematizado / monitorizado e, portanto, pouco acreditado.

Estas e outras explicações (como é o caso da orientação dos conteúdos visados na formação dos professores) recaem, na sua origem, sobre o desconhecimento da influencia ambiental no processo de incapaci- 
dade e funcionalidade, onde tal como refere Altman (s/d) a pouca informação existente consiste na sua maioria em experiências pessoais mais do que em dados organizados que permitam identificar padrões de características ambientais barreiras ou facilitadoras. É, pois, hoje uma necessidade iminente a expansão de evidências que suportem a conceptualização dos fatores ambientais e que facilitem a identificação de fatores influentes no processo de participação e inclusão de indivíduos com incapacidade para o desenvolvimento de estratégias que tornam os contextos de aprendizagem efetivamente capazes de responder e celebrar a diversidade humana e funcional.

Assim, neste estudo pretendemos mapear aspetos ambientais influentes no processo de inclusão de crianças/ jovens com incapacidade em contexto educativo. Especificamente, tendo por referência a sistematização de Whiteneck et al. (1997) e a CIF-CJ (OMS, 2007), é procurada resposta às seguintes questões:

- Quais as características ambientais e, especificamente, os produtos e tecnologias que, segundo as equipas educativas, são influentes no funcionamento/ participação de crianças com necessidades adicionais de suporte?

- Em que medida as dimensões ambientais e dos produtos e tecnologias focados pelas equipas variam em função da natureza das incapacidades consideradas?

A escolha das taxonomias de Whiteneck et al. (1997) e da CIF-CJ (OMS, 2007) para enquadrar esta análise, fundamenta-se na ampla e transversal utilização destes dois modelos em diferentes áreas disciplinares e na sua forte presença, especificamente da CIF-CJ, nos discursos relativos aos direitos das pessoas com incapacidade (Hurst, 2003).

\section{Método}

\section{Materiais}

A identificação de dimensões ambientais e produtos e tecnologias considerados influentes no processo de inclusão de crianças com necessidades adicionais de suporte foi realizada através da analise de Programas Educativos Individuais (PEI) desenhados para 170 crianças sinalizadas 
Mónica Silveira-Maia, Pedro Lopes-dos-Santos, Manuela Sanches-Ferreira, Silvia Alves y

para os serviços de educação especial. Nos PEl estiveram, especificamente, sob escrutínio: as descrições do funcionamento/ desempenho dos alunos; os objetivos e estratégias inscritos nas respostas educativas acionadas.

Após autorização expressa da Direção Geral de Inovação e Desenvolvimento Curricular para o desenvolvimento deste estudo, foram contactados, inicialmente, 316 agrupamentos escolares, de Portugal Continental, abrangidos pelas Direções Regionais de Educação de Lisboa e Vale do Tejo, do Norte, do Centro, do Alentejo e do Algarve. A seleção destes agrupamentos obedeceu a processo de escolha aleatória em cada uma das Direções, procurando que fosse respeitada a proporcionalidade numérica entre a quantidade total dos agrupamentos existentes nas várias estruturas regionais. Dentre os contatos realizados, 73 agrupamentos dispuseram-se a proporcionar a recolha da documentação solicitada - cada um disponibilizando para análise os PEI de 2 a 3 alunos, com devido consentimento informado dos encarregados de educação. Foram obtidos um total de 302 PEl, dos quais apenas 170 foram considerados para análise por integrarem quer o processo avaliativo (i.e., a descrição do funcionamento do aluno) quer interventivo (i.e., com especificação das medidas a implementar). Os restantes processos $(n=132)$ encontravam-se incompletos. A fim de assegurar a confidencialidade das informações, foram eliminados quaisquer dados pessoais do aluno ou que permitissem identificar a escola. A cada processo, foi atribuído um código numérico.

Os PEl examinados diziam respeito a 100 alunos do sexo masculino $(58.8 \%)$ e a 70 do sexo feminino $(41.2 \%)$. As suas idades estavam compreendidas entre os 6 e os 22 anos, com média situada nos 11.98 anos $(D P=3.04)$. Parte substancial dos $\mathrm{PEI}(77.65 \%, \mathrm{n}=132)$ reportam-se ao $1^{\circ}$ e $2^{\circ}$ ciclos de escolaridade.

Quanto à natureza das incapacidades, 91 referiam-se a alterações de natureza predominantemente cognitiva, 28 comportamentais ou sociais, 14 motoras, 10 sensoriais e 27 apresentavam multideficiência. O diagnóstico médico e a proeminência de determinados domínios de funcionamento nos perfis de desempenho dos alunos (especificamente funções mentais, sensoriais, da fala, e neuromusculoesqueléticas) foram tomados em consideração para a categorização dos casos em diferentes grupos/ tipos de incapacidade, especificamente, enquadraram-se nas categorias:

- cognitiva, quando as descrições recaiam sobre as funções men- 
Dimensões ambientais e produtos e tecnologias influentes na participação e inclusão de alunos com incapacidade

Mónica Silveira-Maia, Pedro lopes-dos-Santos, Manuela Sanches-Ferreira, Silvia Alves y ANA PARADA

tais e incluíam diagnósticos como incapacidade intelectual, atraso global de desenvolvimento, síndrome de Down;

- motora, quando as alterações incidiam sobre as funções neuromusculoesqueléticas, incluindo diagnósticos como paralisia cerebral, atrofia muscular progressiva, espinha bífida;

- multideficiência, quando se verificava associação de alterações nas funções mentais e neuromusculoesqueléticas e/ou sensoriais, incluindo diagnósticos como síndrome Polimalformativo, síndrome Alcoólico-fetal, síndrome de Prader-Willi, e outros (nomeadamente paralisia cerebral, síndrome de Down, quando revelavam simultaneidade de deficiências cognitivas e motoras);

- comportamental/ social, quando se reportam alterações psicossociais e psicomotoras, incluindo diagnósticos como perturbações do espetro do autismo, perturbação de hiperatividade com défice de atenção;

- sensorial, na presença de alterações do domínio sensorial, com diagnóstico de deficiência auditiva ou visual.

Os PEI foram projetados por equipas constituídas pelo professor de educação especial e pelo diretor de turma/docente titular de turma, contando, frequentemente, com a colaboração de profissionais como psicólogos, terapeutas da fala, terapeutas ocupacionais, e intérpretes/formadores de Língua Gestual Portuguesa.

\section{Processo analítico}

Os conteúdos dos PEI foram objeto de análise de conteúdo, procedendose à identificação de unidades de significado e sua categorização dedutiva nas dimensões ambientais propostas por Whiteneck et al. (1997) e nos domínios ambientais apresentados pela CIF-CJ (OMS, 2007).

As unidades de significado foram identificadas ao nível da frase ou do segmento de frase sempre que os seus conteúdos se reportavam a fatores extrínsecos capazes de influenciar o comportamento/ funcionamento do aluno. O processo de condensação do significado e de categorização das unidades de significado, exemplificado na tabela 3, envolveu uma interpretação do significado implícito ao texto numa análise de conteúdo, predominantemente, latente (Graneheim \& Lundman, 2004). No processo de categorização por referência à CIF-CJ, socorremo-nos também de 
Dimensões ambientais e produtos e tecnologias influentes na participação e inclusão de alunos com incapacidade

Mónica Silveira-Maia, Pedro lopes-dos-Santos, Manuela Sanches-Ferreira, Silvia Alves y

uma análise de conteúdo manifesta, sempre que as descrições faziam já uso da sua taxonomia (e.g., "os produtos e tecnologias para a educação são facilitadores - /Produtos e Tecnologias (para a educação)/"'). Dentro de cada esquema de categorização, a ligação dos conteúdos a cada dimensão obedeceu ao principio de mutua exclusividade, isto é, nenhuma unidade de significado foi situada em duas categorias simultaneamente.

Tabela 3. Exemplo do processo de condensação do significado e de categorização.

\begin{tabular}{|c|c|c|c|}
\hline $\begin{array}{l}\text { Unidade de Signifi- } \\
\text { cado }\end{array}$ & $\begin{array}{l}\text { Condensação da } \\
\text { unidade de signi- } \\
\text { ficado e ligação } \\
\text { às categorias de } \\
\text { Whiteneck et al }\end{array}$ & $\begin{array}{l}\text { Unidade de } \\
\text { Significado }\end{array}$ & $\begin{array}{l}\text { Condensação da uni- } \\
\text { dade de significado e } \\
\text { ligação às categorias } \\
\text { da CIF-CJ }\end{array}$ \\
\hline $\begin{array}{l}\text { "a sala de aula tem } \\
\text { espaços amplos para } \\
\text { se mover" }\end{array}$ & $\begin{array}{l}\text { Acesso físico e/ou } \\
\text { aspetos arquitetó- } \\
\text { nicos } \rightarrow \text { Acessibi- } \\
\text { lidade }\end{array}$ & $\begin{array}{l}\text { "uso de manuais } \\
\text { ou softwares } \\
\text { educativos adap- } \\
\text { tados" }\end{array}$ & $\begin{array}{l}\text { Produtos/ método } \\
\text { adaptados para a } \\
\text { aquisição de conheci- } \\
\text { mentos } \rightarrow \text { Produtos e } \\
\text { tecnologias de apoio } \\
\text { (para educação) }\end{array}$ \\
\hline $\begin{array}{l}\text { "segmentação das } \\
\text { instruções" }\end{array}$ & $\begin{array}{l}\text { Adequação/ modifi- } \\
\text { cação das tarefas } \rightarrow \\
\text { Adaptabilidade }\end{array}$ & $\begin{array}{l}\text { "regulação da luz/ } \\
\text { som às necessi- } \\
\text { dades sensoriais } \\
\text { do aluno" }\end{array}$ & $\begin{array}{l}\text { Nível ou quantidade } \\
\text { de energia emitida } \\
\rightarrow \text { Ambiente natural } \\
\text { (Intensidade da luz) }\end{array}$ \\
\hline $\begin{array}{l}\text { "ter um quadro de } \\
\text { comunicação" }\end{array}$ & $\begin{array}{l}\text { Disponibilidade e } \\
\text { fornecimento de } \\
\text { serviços/ recursos } \\
\rightarrow \text { Disponibilidade } \\
\text { de Recursos }\end{array}$ & $\begin{array}{l}\text { "apoio na exe- } \\
\text { cução de tarefas } \\
\text { por parte de um } \\
\text { par-tutor" }\end{array}$ & $\begin{array}{l}\text { Apoio físico ou } \\
\text { emocional } \rightarrow \text { Apoio e } \\
\text { relacionamentos (de } \\
\text { conhecidos, pares (...) } \\
\text { membros da comuni- } \\
\text { dade) }\end{array}$ \\
\hline $\begin{array}{l}\text { "o seu envolvimen- } \\
\text { to nas tarefas é } \\
\text { reforçado positiva- } \\
\text { mente pelos pares e } \\
\text { professor" }\end{array}$ & $\begin{array}{l}\text { Atitudes que podem } \\
\text { desencorajar ou fa- } \\
\text { cilitar a integração } \\
\text { na comunidade } \rightarrow \\
\text { Suporte Social } \\
\end{array}$ & $\begin{array}{l}\text { "altas expecta- } \\
\text { tivas da família } \\
\text { em relação ao } \\
\text { seu envolvimen- } \\
\text { to académico" }\end{array}$ & $\begin{array}{l}\text { Atitudes influenciam } \\
\text { o comportamento } \\
\text { individual } \rightarrow \text { Atitudes } \\
\text { (da família próxima) }\end{array}$ \\
\hline $\begin{array}{l}\text { "o projeto educativo } \\
\text { é planeado tendo } \\
\text { em consideração o } \\
\text { processo inclusivo } \\
\text { dos alunos com } \\
\text { necessidades educa- } \\
\text { tivas especiais" }\end{array}$ & $\begin{array}{l}\text { Politicas e regu- } \\
\text { lamentos institu- } \\
\text { cionais garantem } \\
\text { a igualdade de } \\
\text { oportunidades } \rightarrow \\
\text { Equidade }\end{array}$ & $\begin{array}{l}\text { "ter o apoio de } \\
\text { profissionais } \\
\text { como o tera- } \\
\text { peuta revela-se } \\
\text { facilitador" }\end{array}$ & $\begin{array}{l}\text { Serviços que propor- } \\
\text { cionam benefícios } \rightarrow \\
\text { Serviços, sistemas e } \\
\text { politicas (de educação } \\
\text { especial e formação } \\
\text { profissional) }\end{array}$ \\
\hline
\end{tabular}

Dentre as menções a Produtos e Tecnologias procurou-se discriminar aqueles atinentes ao: consumo pessoal; vida diária; mobilidade e trans- 
Dimensões ambientais e produtos e tecnologias influentes na participação e inclusão de alunos com incapacidade

Mónica Silveira-Maia, Pedro Lopes-dos-Santos, Manuela Sanches-Ferreira, Silvia Alves y ANA PARADA

porte pessoal; comunicação; educação; cultura, atividades recreativas e desportivas; e arquitetura, construção, materiais e tecnologia arquitetónicas.

Este exercício de categorização e a contagem do número de unidades de significado alocada a cada categoria, permitiu transformar a informação qualitativa em dados quantitativos passíveis de serem processados através de técnicas estatísticas. Assim, a fim de aferirmos o grau de influencia - a partir da média de menções - de cada dimensão no processo de inclusão, usámos a Multianálise da Variância (MANOVA) para medidas repetidas. Quando rejeitada a hipótese nula, com vista a localizar os efeitos significativos, foram testadas diferenças em cada par de variáveis através do teste post hoc de Bonferroni.

Por constituírem grupos com tamanhos amostrais muito distintos e por não se verificar, dentro de cada grupo, o pressuposto de normalidade em relação às variáveis em foco, optamos por usar medianas e a amplitude interquartil (AIQ) para fazer a descrição das referências em função do tipo de incapacidade. Na análise inferencial recorremos aos testes não paramétricos de Kruskal-Wallis e de Mann-Whitney para comparação entre grupos de incapacidade e, dentro de cada grupo, as variações foram analisadas através do teste de Friedman a par do teste de Wilcoxon.

\section{Fiabilidade}

Para avaliar a fiabilidade intercodificador, procedeu-se à codificação independente por parte de três investigadores (autores deste artigo) de uma subamostra de $60 \mathrm{PEI}$. Na ligação das unidades de significado à CIF-CJ, a média de coeficientes de Kappa obtidos variou entre .68 e .89, ultrapassando o nível de .61 que segundo Landis e Koch (1977) representa concordância substancial. No que respeita à fiabilidade na ligação das unidades de significado às cinco dimensões ambientais de Whiteneck et al (1997), usou-se o coeficiente de correlação de Pearson. Os coeficientes obtidos oscilaram entre .58 e .92 - representando, segundo Dancey e Reidy (2005), valores de associação moderados e fortes. 


\section{Resultados}

\section{Dimensões Ambientais}

Nos PEl foram identificados um total de 2876 unidades de significado, representando uma média de $16.92(D P=9.47)$ referências a aspetos ambientais em cada processo. Globalmente, a média de referências diferiu entre dimensões, $F(4,166)=115.29 ; p<.001, p 2=.41$, havendo um enfoque em questões de suporte social, disponibilidade e adaptabilidade, significativamente superior àquele verificado no que respeita à acessibilidade e equidade. Dentre estas três dimensões, o suporte social e a disponibilidade são mais referidos comparativamente às questões de adaptabilidade $(p=.004 ; p<.001)$.

A variação no enfoque concedido às cinco dimensões (tabela 4) verificou-se, conforme mostra o teste de Friedman, dentro de todos os grupos de incapacidade. De um modo transversal às incapacidades cognitiva, motora e multideficiência os aspetos de suporte social e disponibilidade assumem maior relevo. À semelhança do globalmente aferido, nas incapacidades cognitivas e nos casos de multideficiência estas duas dimensões são significativamente mais abordadas do que as questões de adaptabilidade $(Z=-6.16, p<.001 ; Z=-3.13, p=.002)$. O mesmo não acontece nas incapacidades motoras onde a abordagem à disponibilidade e adaptabilidade não difere com significância estatística. Nas incapacidades comportamentais/ sociais e sensoriais a adaptabilidade passa a assumir protagonismo, conjuntamente com o suporte social. No grupo das incapacidades sensoriais o número de referências a questões da adaptabilidade chega a diferir com significância estatística da dimensão de disponibilidade $(Z=-2.44, p=.02)$. 
Dimensões ambientais e produtos e tecnologias influentes na participação e inclusão de alunos com incapacidade

Mónica Silveira-Maia, Pedro Lopes-dos-Santos, Manuela Sanches-Ferreira, Silvia Alves y Ana Parada

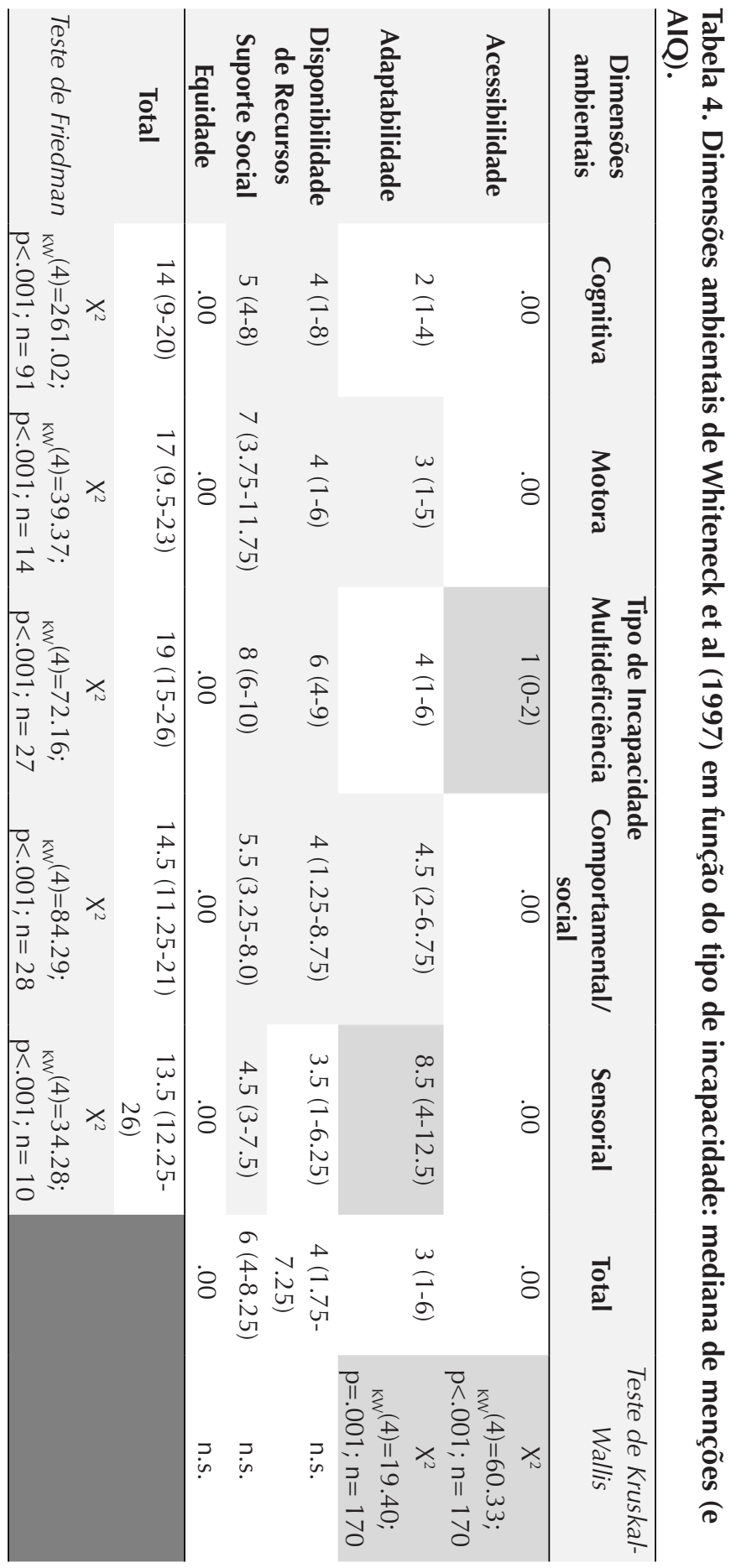


Entre os diferentes grupos de incapacidade, conforme revela o teste de Kruskal-Wallis, existem variações na expressão de aspetos de acessibilidade e adaptabilidade. A acessibilidade assume maior relevo nos casos de multideficiência, quando comparado com incapacidades cognitivas $(U=611.5, W=4797.5, p<.001)$, comportamentais/ sociais $(U=182$, $W=588, p<.001)$ e sensoriais $(U=65, W=120, p=.006)$. Já as questões de adaptabilidade são mais referidas nas incapacidades sensoriais, comparativamente aos grupos cognitivo $(U=138, W=4324, p<.001)$, motor $(U=19.5, W=124.5, p=.003)$ e de multideficiência $(U=64, W=442$, $p=.01)$.

\section{Produtos e Tecnologias}

Foram enquadradas 2871 unidades de significado na taxonomia ambiental da CIF-CJ - corporizando uma média de 16.89 ( $D P=9.43)$ menções a fatores ambientais em cada processo. A atenção dedicada a cada domínio ambiental da CIF-CJ é globalmente diferente, $F(4,166)=155.66$; $p<.001, \eta_{p} 2=.48$, havendo predominância, estatisticamente significativa, de menções a produtos e tecnologias, seguido da abordagem ao apoio e relacionamentos. Os serviços, sistemas e politicas, e as atitudes são pouco abordados; e as menções a aspetos do ambiente natural são praticamente inexistentes.

Esta tendência verifica-se dentro de cada um dos grupos de incapacidade, com os produtos e tecnologias a serem, de maneira estatisticamente significativa, mais abordados que todos os restantes, seguido pelo domínio do apoio e relacionamentos que se demarca também com significância estatística das atitudes, serviços/ sistemas e politicas, e ambiente natural (tabela 5). 
Dimensões ambientais e produtos e tecnologias influentes na participação e inclusão de alunos com incapacidade

Mónica Silveira-Maia, Pedro Lopes-dos-Santos, Manuela Sanches-Ferreira, Silvia Alves y ANA PARADA

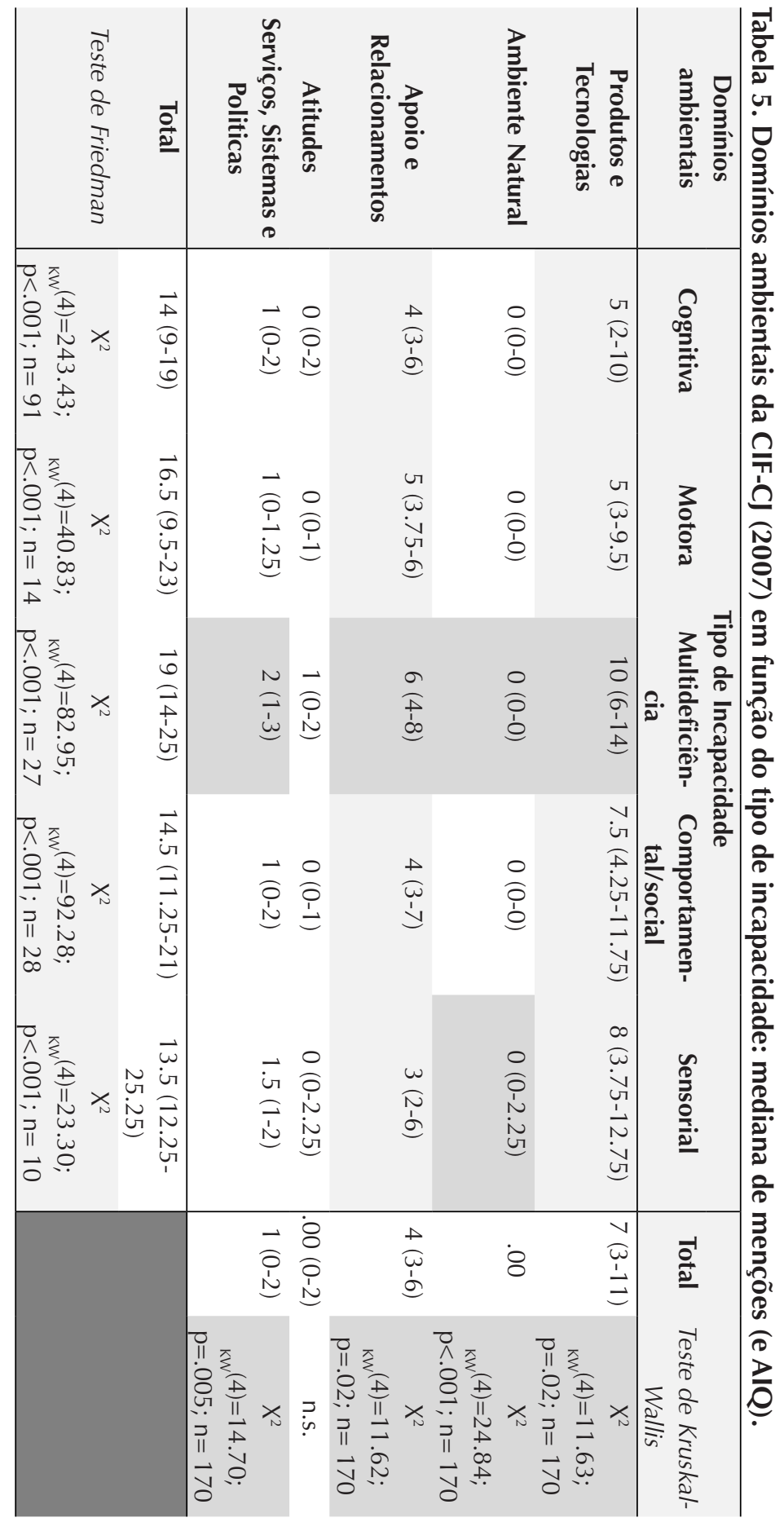


Entre os diferentes grupos de incapacidade, conforme revela o teste de Kruskal-Wallis, existem variações no enfoque concedido a todos os domínios, à exceção dos aspetos atitudinais. A globalidade dos domínios parecem assumir maior expressão nos casos de multideficiência, demarcando-se das incapacidades cognitivas (em relação aos produtos e tecnologias, $U=759, W=4945, p=.003$, ao ambiente natural, $U=1105.5$, $W=5291.5, p=.01$, ao apoio e relacionamentos $U=772, W=4958, p=.003$ e aos serviços, sistemas e politicas, $U=706.5, W=4892.5, p<.001)$, das incapacidades comportamentais/sociais (serviços, sistemas e politicas, $U=240.5, W=646.5, p=.02$ ) e das sensoriais (apoio e relacionamentos, $U=68, W=123, p=.02$ ). Já as questões relativas ao ambiente natural assumem maior relevo nas incapacidades sensoriais, comparativamente aos grupos cognitivo $(U=138, W=4324, p<.001)$, motor $(U=19.5, W=124.5$, $p=.003)$ e de comportamental/social $(U=64, W=442, p=.01)$.

Dentre os produtos e tecnologias (tabela 6) destacam-se, em todos os grupos de incapacidade, aqueles concernentes à educação, especificamente materiais, métodos e tecnologias adaptados ou especialmente concebidos (e.g., computadores e softwares adaptados, uso de objetos tangíveis, método global para ensino da leitura). 
Dimensões ambientais e produtos e tecnologias influentes na participação e inclusão de alunos com incapacidade

Mónica Silveira-Maia, Pedro Lopes-dos-Santos, Manuela Sanches-Ferreira, Silvia Alves y ANA PARADA

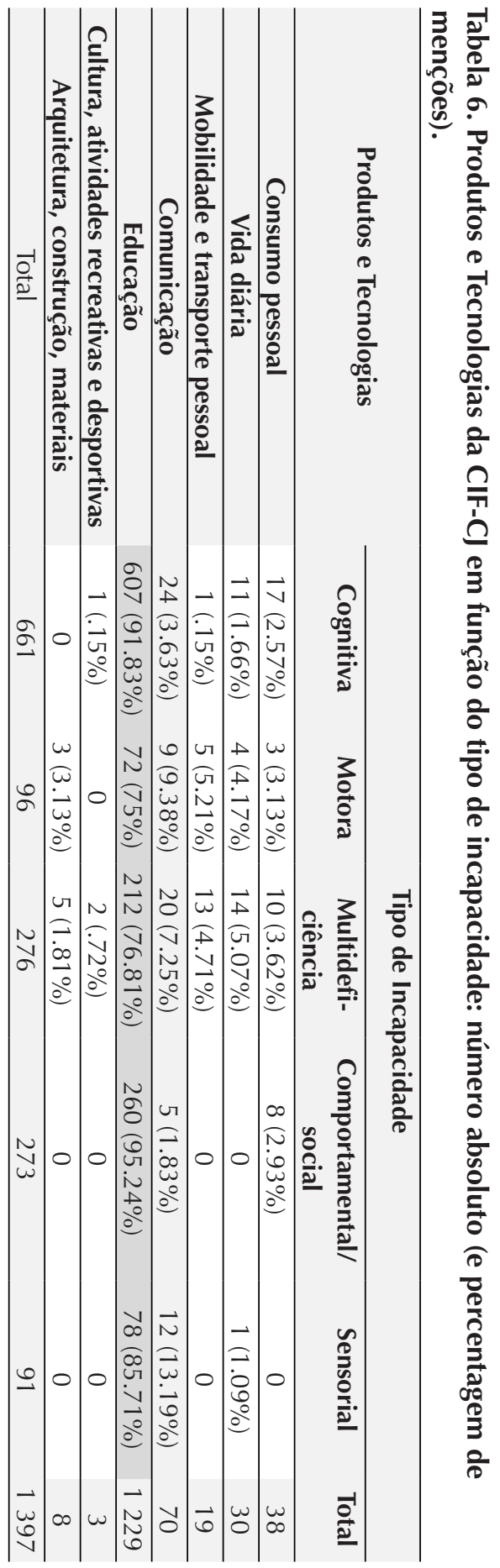


Mónica Silveira-Maia, Pedro Lopes-dos-Santos, Manuela Sanches-Ferreira, Silvia Alves y

Os produtos e tecnologias para a comunicação também surgem com alguma frequência, incluindo desde placas de comunicação até engrossadores de cabo para escrita manual.

\section{Discussão}

Este artigo teve como objetivo identificar e descrever aspetos ambientais influentes no processo de inclusão de alunos com incapacidade, inscrevendo-se num quadro de valores e de conhecimentos que atribui à manipulação/ adequação de variáveis do meio um papel central no cumprimento dos requisitos de equidade e justiça social que definem hoje uma educação de qualidade (e.g., Noreau \& Boschen, 2010; Florian, 2014; Silveira-Maia et al., 2016)

A partir dos processos de avaliação e intervenção contemplados nos PEI, os resultados sugerem que o suporte social (e.g., expectativas, atitudes, comportamentos de apoio), a disponibilidade (e.g., distribuição e alocação de serviços, recursos, materiais) e a adaptabilidade (e.g., adequação/ ajustamento de métodos de ensino, tarefas e materiais) são, na globalidade dos casos, as dimensões mais consideradas/referenciadas pelas equipas educativas no apoio à funcionalidade e participação dos alunos com incapacidade. Esta tendência é verificada na abordagem a cada grupo de incapacidade, onde o suporte social e a disponibilidade representam as dimensões mais abordadas nos casos de incapacidade cognitiva e de multideficiência; e onde nos grupos de incapacidade motora, comportamental/ social e sensorial, a adaptabilidade ganha igual protagonismo.

Quando analisadas possíveis diferenças, entre grupos, na expressão das cinco dimensões ambientais, observou-se maior saliência de questões de adaptabilidade nas incapacidades sensoriais (e.g., esquematização, uso de concretização, ajustamento de manuais). Já os aspetos de acessibilidade são equacionados, sobretudo, nos quadros de multideficiência (e.g., existência de rampas, elevadores, largura dos corredores). Não obstante a sua aparente proeminência nos casos de multideficiência, a acessibilidade, acompanhada da equidade, são dimensões pouco presentes nos PEI. Tal parece colocar a acessibilidade e equidade num plano de concretizações relacionado com uma cultura de escola e/ou com esforços de cariz tendencialmente político/ administrativo, que se 
Dimensões ambientais e produtos e tecnologias influentes na participação e inclusão de alunos com incapacidade

Mónica Silveira-Maia, Pedro Lopes-dos-Santos, Manuela Sanches-Ferreira, Silvia Alves y ANA PARADA

veem vertidos nos PEI através da mobilização de aspetos de disponibilidade, adaptabilidade e suporte social.

Este enquadramento no referencial Whiteneck et al. (1997) encontra correspondência na distribuição/ ligação de referências à taxonomia da CIF-CJ (OMS, 2007), onde se podem ver refletidas as dimensões de disponibilidade e adaptabilidade na frequente abordagem aos Produtos e Tecnologias (i.e., métodos/equipamentos/materiais); e onde o suporte social é transparecido no destaque concedido ao domínio do Apoio e Relacionamentos (i.e., apoio prático físico ou emocional).

Quando analisada a distribuição de referências pelos diferentes grupos de incapacidades, os resultados mostram que a globalidade dos domínios ambientais da CIF-CJ se vê mais mobilizado (i.e., referenciado) nos casos de multideficiência - incluindo os aspetos relacionados, não só, com os materiais e serviços, mas também, com o apoio emocional e físico. Outra variação encontra-se na referência a aspetos do Ambiente Natural, assumindo destaque nas incapacidades sensoriais (e.g., aspetos de luminosidade, ruido) comparativamente aos restantes grupos. Importa notar, contudo, que este domínio a par das Atitudes, e Serviços, Sistemas e Politicas é pouco mencionado à semelhança do que acontece, como já discutimos, com as dimensões de equidade e acessibilidade. Quando escrutinadas as menções aos produtos e tecnologias, os métodos/ equipamentos/ materiais educativos especialmente concebidos ou adaptados assumem centralidade em todos os grupos de incapacidade.

Recuperando os conceitos macro, meso e microssistémico sobre os quais se equacionam os diferentes planos operacionais da inclusão, traçaremos três níveis de reflexões/implicações práticas a partir dos resultados.

A nível micro - i.e., de sala de aula -, os resultados sugerem a influencia determinante da disponibilidade e adaptabilidade de produtos e tecnologias para a educação, onde se incluem exemplos como o uso de manuais, computadores e softwares adaptados. Os métodos/ produtos relacionados com a comunicação surgem também mencionados com alguma frequência, sugerindo a sua influencia emergente nos processos de inclusão, nomeadamente nas incapacidades sensoriais, motoras e na multideficiência. O desenvolvimento de materiais didáticos e outros produtos que atendam às características de funcionamento e necessidades adicionais decorrentes dos diferentes tipos/grupos de incapacidade ou que, sob a perspetiva de um desenho universal, contemplem flexibi- 
Mónica Silveira-Maia, Pedro Lopes-dos-Santos, Manuela Sanches-Ferreira, Silvia Alves y

lidade no seu uso parece, deste modo, constituir um aspeto central nos processos de inclusão. De facto, estes resultados vão de encontro ao atual protagonismo das tecnologias de comunicação e informação (TIC) em educação, desempenhando um papel fundamental no desenvolvimento de currículos flexíveis e facilitando a implementação de um processo de ensino-aprendizagem centrado nos estilos de aprendizagem/ funcionamento de cada aluno (EADSNE, 2013).

A nível meso - i.e., serviços da escola e comunidade -, é transparecida a importância do desenvolvimento de uma cultura de inclusão vertida na distribuição / alocação de recursos materiais (i.e., disponibilidade) e no desenvolvimento de redes de apoio e relacionamentos (i.e., suporte social). Esta cultura de escola inclusiva, onde se torna iminente a rutura com um modelo do cuidador - onde a capacitação / envolvimento dos alunos com incapacidades é visto como um encargo de profissionais especializados- para se estabelecer um modelo de redes sociais de apoio, onde a inclusão social é uma responsabilidade de todos, tem sido uma perspetiva aclamada quer no domínio dos valores e direitos humanos e.g., Convenção sobre os Direitos das Pessoas com Incapacidade (NU, 2006) - quer na conceção dos modelos de suporte que hoje orientam a avaliação e a intervenção sobre indivíduos com incapacidade (e.g., Shalock et al., 2010). Uma das linhas de investigação e ação (e.g., Tavares, 2011; Godeau et al., 2010) que mais notoriamente resulta dessa perspetiva, têm sido a abordagem interventiva sobre as atitudes dos alunos com desenvolvimento típico face aos pares com incapacidades, onde se consideram os programas de sensibilização para a incapacidade ferramentas úteis na "criação, dentro das turmas, de condições de recetividade e de respeito pela diferença" (Alves \& Lopes-dos-Santos, 2013, p. 515).

A nível macro - i.e., quadros legais, políticos e administrativos - destaca-se a necessidade de mobilizar mecanismos de apoio para uma prática sustentada de habilitação ambiental, instigando o desenvolvimento de recursos, conhecimentos e competências para avaliar, intervir e monitorizar a manipulação/ adequação de variáveis ambientais. Neste domínio, as dimensões de disponibilidade e adaptabilidade de produtos e tecnologias, bem como o suporte social (apoio e relacionamentos) são conteúdos ambientais que merecem ser, não só, melhor conhecidos mas, sobretudo, compreendidos no que concerne às suas implicações sobre a funcionalidade e participação social. Tais desígnios implicam necessariamente o domínio da investigação - no desenvolvimento de 
Dimensões ambientais e produtos e tecnologias influentes na participação e inclusão de alunos com incapacidade

Mónica Silveira-Maia, Pedro lopes-dos-Santos, Manuela Sanches-Ferreira, Silvia Alves y ANA PARADA

instrumentos de avaliação e de evidências empíricas capazes de retratar a influencia do ambiente nas vidas das pessoas com incapacidade (Whiteneck et al., 2004); e a formação das equipas educacionais para integrar, de modo efetivo, a componente ambiental nos processos de avaliação e intervenção de alunos com necessidades adicionais de suporte - aspeto ainda pouco operacionalizado nos $\mathrm{PEl}$, cujos conteúdos continuam ainda essencialmente voltados para variáveis pessoais (Janney \& Snell, 2006).

\section{Referências bibliográficas}

Alves, S., \& Lopes-dos-Santos, P. (2013). Bringing disability awareness into the general curriculum. In J. A. Pacheco et al. (Orgs.), Proceedings of the European conference on curriculum studies "Future directions: uncertainty and possibility" (pp. 514-518). Paper presented at European conference on curriculum studies "Future directions: uncertainty and possibility". Braga: University of Minho.

Altman, B. (n.d.). Measuring Environmental Factors in an International Context: A Social System Perspective for an Extended Question Set. Retrieved from: https://www.cdc. gov/nchs/data/washington_group/meeting11/measuring_environmental_factors_draft_paper.pdf

Bronfenbrenner, U. (1979). The Ecology of Human Development: Experiments by nature and design. Cambridge: Harvard University Press.

Dancey, C., \& Reidy, J. (2006). Estatística Sem Matemática para Psicologia:Usando SPSS para Windows. Porto Alegre: Artmed.

Decreto-Lei n. ${ }^{o}$ 3/2008 - Public Law 2/2008. (2008). Diário da República - Ministério da Educação Serie N. ${ }^{\circ}$ 4-7 Janeiro: 154-164.

European Agency for Development in Special Needs Education. (2013). Information and Communication Technology for Inclusion - Developments and Opportunities for European Countries. Odense: European Agency for Development in Special Needs Education.

European Agency for Development in Special Needs Education. (2011). Participation in Inclusive Education - A Framework for Developing Indicators. Odense: European Agency for Development in Special Needs Education.

Florian, L. (2014). What counts as evidence of inclusive education? European Journal of Special Needs Education, 29 (3), 286-294. doi: 10.1080/08856257.2014.933551

Fougeyrollas, P., \& Beauregard, L. (2011). Disability: and interactive person-environment social creation. In: G.L. Albrecht, K.D. Seelman, \& M. Bury (Eds.), Handbook of disability studies (pp. 171-94). Thousand Oaks: Sage Publications.

Graneheim, U.H., Lundman, B. (2004). Qualitative content analysis in nursing research: concepts, procedures and measures to achietrustworthiness. Nurse Educ Today, 24, 105-112. 
Dimensões ambientais e produtos e tecnologias influentes na participação e inclusão de alunos com incapacidade Mónica Silveira-Maia, Pedro Lopes-dos-Santos, Manuela Sanches-Ferreira, Silvia Alves y ANA PARADA

Godeau, E., Vignes, C., Sentenac, M., Ehlinger, V., Navarro, F., Grandjean, H., \& Arnaud, C. (2010). Improving attitudes towards children with disabilities in a school context: A cluster randomized intervention study. Dev Med Child Neurol, 52(10), 236-242. doi: 10.1111/j.1469-8749.2010.03731.x

Hollenweger, J. (2011). Development of an ICF-based eligibility procedure for education in Switzerland. BMC Public Health, 11 (4), S7.

Hurst, R. (2003). The international disability rights movement and the ICF. Disabil. Rehabil, 25, 572-576.

Janney, R., Snell, M. (2006). Modifying Schoolwork in Inclusive Classrooms. Theory into Practice, 45 (3), 215-223.

Kyriazopoulou, M., \& Weber, H. (2009). Development of a set of indicators - for inclusive education in Europe. Odense: European Agency for Development in Special Needs Education.

Landis, J.R., \& Koch, G.G. (1977). The measurement of observer agreement for categorical data. Biometrics, 33, 159-174.

Lewin, K. (1935). Environmental forces in child behavior and development: a dynamic theory of personality. New York: McGraw-Hill Book Co.

Maxwell, G., \& Granlund, M. (2011). How are conditions for participation expressed in education policy documents? A review of documents in Scotland and Sweden. European Journal of Special Needs Education, 2(26), 251-272.

Noreau, L., \& Boschen, K. (2010). Intersection of Participation and Environmental Factors: A Complex Interactive Process. Arch Phys Med Rehabil, 91(1), S44-S53.

Norwich, B. (2014). Changing policy and legislation and its effects on inclusive and special education: a perspective from England. British Journal of Special Education, 41(4), 403-425. doi: 10.1111/1467-8578.12079

Organização Mundial de Saúde. (2007). Classificação Internacional de Funcionalidade, Incapacidade e Saude, versão para Crianças e Jovens. Geneva: Organização Mundial de Saúde.

Organização Mundial de Saúde. (2001). Classificação Internacional de Funcionalidade, Incapacidade e Saude. Geneva: Organização Mundial de Saúde.

Reindal, S. (2010). What is the purpose? Reflections on inclusion and special education from a capability perspective. European Journal of Special Needs Education, 25 (1), 1-12. doi: 10.1080/08856250903450806

Rosenberg, L., Ratzon, N., Jarus, T., \& Bart, O. (2011). Perceived environmental restrictions for the participation of children with mild developmental disabilities. Child: care, health and development, 38 (6), 836-843. doi:10.1111/j.1365-2214. 2011.01303.x

Sanches-Ferreira, M., Simeonsson, R., Silveira-Maia, M., \& Alves, S. (2015). Evaluating implementation of the International Classification of Functioning, Disability and Health in Portugal's special education law. International Journal of Inclusive Education, 19(5), 457-468. doi: 10.1080/13603116.2014.940067

Sanches-Ferreira, M., Simeonsson, R., Silveira-Maia, M., Alves, S., Tavares, A., \& Pinheiro, S. (2013). Portugal's Special Education Law: Implementing the International Classification of Functioning, Disability and Health in Policy and Practice. Disability \& Rehabilitation, 35 (10), 868-873. doi:10.3109/09638288.2012.708816 
Dimensões ambientais e produtos e tecnologias influentes na participação e inclusão de alunos com incapacidade

Mónica Silveira-Maia, Pedro lopes-dos-Santos, Manuela Sanches-Ferreira, Silvia Alves y

ANA PARADA

Schalock, R. L., Borthwick-Duffy, S., Bradley, V., Buntix, W. H. E., Coulter, D. L., Craig, E. P. M., \& Yeager, M. H. (2010). Intellectual disability: Definition, classification, and systems of support (11th ed.). Washington, DC: American Association on Intellectual and Developmental Disabilities.

Sen, A. K. (1992). Inequality reexamined. New York: Harvard University.

Shogren, K., Wehmeyer, M., Seo, H., Thompson, J., Schalock, R., Hughes, C., Little, T., \& Palmer, S. (2016). Examining the Reliability and Validity of the Supports Intensity Scale-Children's. Version in Children With Autism and Intellectual Disability. Focus on Autism and Other Developmental Disabilities. doi: ·10.1177/1088357615625060

Silveira-Maia, M., Lopes-dos-Santos, P., \& Sanches-Ferreira, M. (2016). How the use of the International Classification of Functioning, Dsablity and Health, version for children and youth (ICF-CY) changed the Individualized Education Programs (IEP) in Portugal. International Journal of Inclusive Education, 21(5). doi: 10.1080/13603116.2016.1218950

Simeonsson, R.J., Simeonsson, N.E., \& Hollenweger J. (2008). International Classification of Functioning, Disability and Health for Children and Youth: A Common Language for Special Education. In L. Florian \& M. McLaughlin (Eds.), Disability Classification in Education: Issues and Perspectives (pp. 207-217). California: Corwin Press.

Tavares, W. (2011). An evaluation of the Kids are Kids disability awareness program: Increasing social inclusion among children with physical disabilities. J Soc Work Disabil Rehabil, 10(1), 25-35. doi: 10.1080/1536710X.2011.546296

Terzi, L. (2014). Reframing inclusive education: Educational equality as capability equality. Cambridge Journal of Education, 44, 479-493.

Terzi, L. (2004). The social model of disability: A philosophical critique. Journal of Applied Philosophy, 21 (2), 141-57.

Whiteneck, G., Meade, M., Dijkers, M., Tate, D., Bushnik, T., \& Forchheimer, M. (2004). Environmental factors and their role in participation and life satisfaction after spinal cord injury. Arch Phys Med Rehabil, 85, 1793-803.

Whiteneck, G.G., Fougeyrolles, P., Gerhart, K.A. (1997). Elaborating the Model of Disablement. In M. Fuhrer (Ed.), Assessing Medical Rehabilitation Practices: The Promise of Outcomes Research. Baltimore: Paul H. Brooks Publishing Co.

\section{Nota:}

Este trabalho foi apoiado pela Fundação para a Ciência e a Tecnologia (FCT) ao abrigo da Bolsa n. ${ }^{\circ}$ SFRH/BD/47417/2008. 